\title{
Median filter algorithm for estimating the threshold of detection on custom protein arrays
}

\author{
David D. Smith ${ }^{1}$, Susan Kovats ${ }^{2}$, Terry D. Lee ${ }^{1}$, and Leticia Cano \\ ${ }^{1}$ City of Hope National Medical Center and Beckman Research Institute, Duarte, CA, \\ ${ }^{2}$ Oklahoma Medical Research Foundation, Oklahoma City, OK, and ${ }^{3} \mathrm{NHLBI}, \mathrm{NIH}$, \\ Bethesda, MD, USA
}

BioTechniques 41:74-78 (July 2006)

doi $10.2144 / 000112204$

We constructed protein arrays according to a titration design to estimate the assay sensitivities over varying concentrations of flu vaccine and human immunoglobulin $G(I g G)$. After imaging, we considered the problem of appropriately distinguishing background noise from foreground signal. We applied the median filter smoothing technique and estimated the differences of the observed signal compared to the smoothed signal. If the absolute value of the difference was large, the feature was easily detectable, indicating that the spot did not blend with its surrounding neighbors. After estimating the residuals, we applied thresholding algorithms to estimate the limits of detection for each assay. At sufficiently large smoothing spans, our median filter approach performed as well or better than visual inspection and two other competing analysis methods. This suggests that a median filter approach has utility in high-throughput arrays where visual inspection is impractical.

\section{INTRODUCTION}

Protein arrays have proven to be valuable tools in a wide variety of settings. The high-throughput features of array technology allow for the screening of thousands of proteins at a time (1-5). However, there are some occasions in immunology when experiments do not easily lend themselves to parallel processing. For example, it is often difficult to isolate an interesting set of proteins for further study when the set of input proteins is unknown. Many effective statistical approaches have been proposed for commercial protein array feature detection, but custom experiments remain in which there are too many unknown quantities for a conventional feature detection analysis.

In our protein expression studies, we were faced with analyzing a series of custom, hand-spotted arrays. One of our early objectives was to estimate the material concentration at which features were detectable. Our design was a titration design. Our approach for detecting features was to find the concentration where foreground signal was indistinguishable from background noise. We present our results using a two-dimensional (2-D) median filter with varying neighborhoods to separate features from noise, and we compare these results with results from alternative methods of signal detection.

\section{MATERIALS AND METHODS}

\section{Materials}

Influenza virus vaccine was purchased from Sanofi-Aventis (Bridgewater, NJ, USA). Immune Globulin Gammagard ${ }^{\circledR}$ (Baxter International, Deerfield, IL, USA) was used as a source of immunoglobulin $\mathrm{G}$ ( $\mathrm{IgG}$ ). Nitrocellulose membrane with a $0.2 \mu \mathrm{m}$ pore size was purchased from Schleicher \& Schuell (Keene, NH, USA). A multiblot replicator, a multiprint, and a library copier (V\&P Scientific, San Diego, CA, USA) were used to spot the protein arrays. We will refer to these as macroarrays, since the hand-stamped spots were $2.25 \mathrm{~mm}$ apart. The replicator contains 96 pin tools designed to hold $0.1 \mu \mathrm{L}$ fluid by the hanging drop method. A highdensity multiprint holds the nitrocel- lulose membrane in place and contains 16 alignment holes. The alignment holes allow 16 microtiter plates to be spotted onto one membrane. $\mathrm{Cy}^{\mathrm{TM}} 3$ donkey anti-human antibody and horseradish peroxidase (HRP)-donkey anti-human antibody were obtained from Jackson ImmunoResearch (West Grove, PA, USA). ECL Plus ${ }^{\mathrm{TM}}$ with HRP-labeled secondary antibody was obtained from Amersham Biosciences (Piscataway, NJ, USA).

\section{Protein Macroarray Construction}

Different concentrations of flu vaccine and human $\operatorname{IgG}$ were spotted onto arrays to determine the limits of detection of two visualization techniques: $\mathrm{Cy} 3$ directly coupled secondary antibody versus ECL Plus with an HRP-coupled secondary antibody. The two arrays consisted of 16 identical spots of different concentrations of flu vaccine and human IgG. For this study, the 16 replicates will be referred to as a well. Different concentrations of flu vaccine and IgG were used to create a mother plate. Flu concentrations ranged sequentially from 2.5 to $0.000005 \mathrm{ng}$; IgG concentrations ranged sequentially from 25 to $0.000005 \mathrm{ng}$. A figure showing the macroarray layout is included in the Supplementary Material (see Supplementary Figure S1 available online at www.BioTechniques.com). Thirty-six wells contained buffer only as a control set. Samples were diluted in $100 \mu \mathrm{L}$ start buffer then 100 $\mu \mathrm{L}$ Ponceau $\mathrm{S}$ in water. Start buffer consisted of $6 \mathrm{M}$ urea, $0.2 \%$ CHAPS, $100 \mathrm{mM}$ ammonium bicarbonate, $\mathrm{pH}$ 8.0. The pink-colored Ponceau $\mathrm{S}$ was used as a visual check to ensure that each pin tool delivered sample to the membrane. The replicator was dipped into the mother plate and $0.1 \mu \mathrm{L}$ sample was transferred onto a precut nitrocellulose membrane. Macroarrays used in this study were spotted on the same day. After spotting, the arrays were dried under a hood. Manual stamping created an imprint onto the nitrocellulose membrane, which indicated the spot position. These imprints were later used after scanning to assist with gridding. 


\section{Short Technical Reports}

\section{Macroarray Processing}

To prevent cross-contamination, each array was processed in a separate Petri dish. The macroarrays were blocked in $1 \%$ bovine serum albumin (BSA) in Tris-buffered saline (TBS) overnight at $4^{\circ} \mathrm{C}$. After blocking, the arrays were washed twice in TBS and probed with a 1:200 dilution of human sera for $1 \mathrm{~h}$ at room temperature. The macroarrays were then washed two more times in TBS $(20 \mathrm{mM}$ Tris- $\mathrm{HCl}$, $\mathrm{pH}$ 7.5, $500 \mathrm{mM} \mathrm{NaCl}, \mathrm{pH}$ 7.5).

Protein arrays were visualized using either Cy3-labeled secondary antibody or ECL Plus with HRPlabeled secondary antibody. For Cy3 comparison: a 1:800 dilution of Cy3-donkey anti-human was used for detection. For ECL Plus, a 1:100,000 dilution of HRP-donkey anti-human antibody was used for detection, which was developed using $4 \mathrm{~mL}$ of the ECL Plus solution. Blots were imaged on a Typhoon $^{\mathrm{TM}} 9410$ variable mode imager (Amersham Biosciences) using 50 $\mu \mathrm{m}$ resolution. The $\mathrm{Cy} 3$ blots were imaged using a $532 \mathrm{~nm}$ excitation (green), 580BP30 filter, at both PMT 400 and $500 \mathrm{~V}$, respectively. The ECL Plus $^{\mathrm{TM}}$ blots were imaged with a 457 nm excitation (blue), 520BP40 filter, PMT at both 450 and $500 \mathrm{~V}$, respectively. This gave us four images from two arrays, one Cy3 array imaged at 400 and $500 \mathrm{~V}$, and one ECL Plus array imaged at 450 and $500 \mathrm{~V}$.

\section{Spot Imaging}

After imaging, the array spots were scanned and quantitated using ImaGene $^{\mathrm{TM}}$ software (BioDiscovery, El Segundo, CA, USA). There were 96 wells with 16 spots/well, giving us 1536 spots after scanning and gridding. For the gridding, we created a template grid with an array composed of $\mathrm{IgG}$ spots. Then, we overlaid our template grid onto the experimental images. We adjusted the gridding manually because of slight variations in the images.

The data derived from ImaGene included statistical estimates of mean, median, mode, area, and standard deviations for both the foreground and background pixels. Background noise and foreground signal estimates were defined around each individual spot without regard to the location on the plate. The technical details of ImaGene's method of foreground and background quantification may be found in Zhou et al. (6). Threedimensional contour plots showed that the background noise was not uniform across the plate. Specifically, the background error was highest among the areas with the highest concentration of flu and $\mathrm{IgG}$ - coordinates corresponding to A1, E1, F1, and G1. After reading in the data, we subtracted the background mean from foreground mean to obtain a signal intensity for each spot.

\section{Methods}

Our statistical approach was to apply a 2-D median filter to the 1536 signal intensities. The median filter was originally proposed by Tukey (7), but has been used extensively in data smoothing and recently in image analysis $(8,9)$. Moore and Jorgenson proposed using the residuals from a median filter to remove low frequency background drift in a single dimension (10).

In image analysis, the 2-D median filter is effective at attenuating noise. It is particularly useful in cases where it is necessary to protect the smoothed image from the influence of outlier pixels. For a given pixel in a 2-D array, the median filter replaces the pixel's value with the median values of all the pixels within a neighborhood of span $r$. At the edges and corners of an image, $r$ would only extend into the interior of the image. The residual of the median filter is defined as the difference between the imputed value and the original value. If the absolute value of the residual is large, the feature is easily detectable, indicating that the spot did not blend with its surrounding neighbors. For convenience, we considered absolute values of residuals.

We conjectured that a reasonable way to find the threshold for which foreground signal is indistinguishable from background noise is by examining the magnitude of the residuals. In our titration design, we considered using concentrations of material that were easily detectable through concentrations too diluted to differentiate from background. If a majority of the values within the median filter are at or near background, the magnitude of the residuals would be large in cases where the concentration is high.

Areas of high fluorescence were easily distinguishable by the naked eye on the macroarrays, particularly when we inverted the grayscale and increased the contrast of the raw image. Figure 1 shows an example of the modified raw image for an ECL Plus-labeled array at $500 \mathrm{~V}$. Darkest areas correspond to areas of high concentrations of material. For measuring the sensitivity and specificity of the median filter, we considered our naked eye assessment as the gold standard. By visual inspection, we determined the wells in which spots were present and which wells had no detectable spots.

\section{RESULTS AND DISCUSSION}

We began by plotting median filter residuals for varying spans against well coordinates. We considered spans ranging from 2 through 10 . Our algorithm was run on a $\mathrm{PC}$ with a 3.2 $\mathrm{GHz}$ processor with $1 \mathrm{~GB}$ RAM. At spans more than 10 , the median filter algorithm became computationally expensive, taking $35 \mathrm{~s}, 12 \mathrm{~min}$, and 19 
min at spans of 2,10 , and 12 , respectively. The algorithm was coded in Splus ${ }^{\mathrm{TM}}$ (Insightful, Seattle, WA, USA), an interpretive statistical programming language (note that Splus is not as efficient in running iterative algorithms as a lower-level language like $\mathrm{C} / \mathrm{C}++)$.

As expected, larger spans improved the ability to distinguish between spots that were present or absent. Figure 2 shows the needle plots for the median filter residuals in all four images. The smoothing span for all four plots was 10. For each well on the plate, we plotted the 16 median filter residuals for each spot. The needle plots showed that wells with long lines indicated where spots were detectable. In rows $\mathrm{B}, \mathrm{C}$, and $\mathrm{D}$, where there was no material, the needle plots showed small, random residuals. The magnitude of the noise was dependent on the macroarray preparation. The macroarray with the most detectable range of spots appeared to be the ECL Plus at $500 \mathrm{~V}$ since it has the largest clusters of vertical lines overall.

The advantage of using median filter residuals for signal detection is that they quantified explicitly the features so that algorithms may be applied to classify spots as present or absent. Our signal detection procedure differs from feature detection in commercially available microarrays because it is more general and it depends simply on intensity data. Commercial microarray manufacturers have historically tailored their detection algorithms to the physical properties of their materials and spot characteristics. An open question is whether commercial array spot detection would improve if an algorithm like the median filter was integrated into manufacturers' software.

Supplementary Table S1 shows the comparison between the numbers of spots that the median filter residuals classified as present, versus the wells with spots that were detectable by the naked eye. We see that wells considered to be on the edge of detection by the naked eye were labeled as misclassified by the median filter residuals, even though in some cases the majority of spots in those wells had values above the numerical threshold of detection (e.g., wells E3 and H3). There are also a couple of spots that were detected by

A
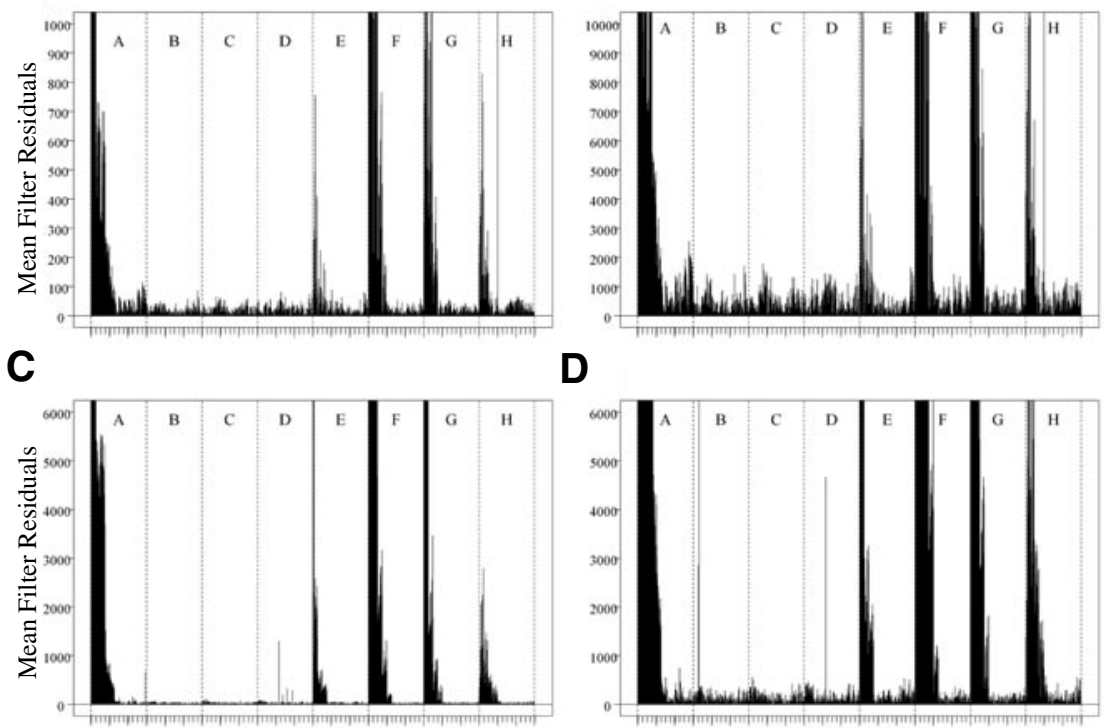

Figure 2. Needle plots with median filter residuals on the y-axis and well coordinates along the $\mathbf{x}$-axis. The long $\mathrm{x}$-axis hash marks are at $0,4,8$, and 12 , with 16 vertical lines within any two adjacent hash marks. (A) Cy3 $400 \mathrm{~V}$; (B) Cy3 $500 \mathrm{~V}$; (C) ECL Plus $450 \mathrm{~V}$; (D) ECL Plus $500 \mathrm{~V}$.

the median filter residuals that were the result of droplets on the plate (wells B2 and D5).

Table 1 shows the sensitivity, specificity, false positive rate, and false negative rates of the median filter residuals after the partition analysis detected the quantitative threshold of detection. As expected, spans that create small neighborhoods for the median filter have more false negative misclassification than spans that create large neighborhoods. We also see that the visual assessment applied to each well may be of limited use for classification purposes, since there are 12/16 unusually large residuals in H3 and $8 / 16$ unusually large residuals in

Table 1. Misclassification Results of the Median Filter Versus the Visual Gold Standard Across Varying Spans and Compared to Two Competing Methods

\begin{tabular}{|c|c|c|c|}
\hline Span & Threshold & $\begin{array}{c}\text { Sensitivity/False Negatives } \\
(\% / \%)\end{array}$ & $\begin{array}{c}\text { Specificity/False Positives } \\
(\% / \%)\end{array}$ \\
\hline 2 & 389 & $56.3 / 43.7$ & $100.0 / 0.0$ \\
\hline 6 & 1040 & $87.5 / 12.5$ & $100.0 / 0.0$ \\
\hline 9 & 1327 & $93.8 / 6.2$ & $100.0 / 0.0$ \\
\hline 10 & 1380 & $100.0 / 0.0$ & $98.8 / 1.2$ \\
\hline \multicolumn{2}{|c|}{ Method } & $\begin{array}{c}\text { Sensitivity/False Negatives } \\
(\% / \%)\end{array}$ & $\begin{array}{c}\text { Specificity/False Positives } \\
(\% / \%)\end{array}$ \\
\hline \multicolumn{2}{|c|}{ Median filter, Span = 10} & $100.0 / 0.0$ & $98.8 / 1.2$ \\
\hline \multicolumn{2}{|c|}{ Dunnett's $t$-test } & $87.5 / 12.5$ & $100.0 / 0.0$ \\
\hline \multicolumn{2}{|l|}{ SAM } & $56.3 / 43.7$ & $71.3 / 28.7$ \\
\hline
\end{tabular}

E3. Both of these wells corresponded to an $\mathrm{IgG}$ concentration of 0.5 , but this concentration was not detectable in other wells with the same concentration, namely F5 and G4 (0/16 and $4 / 16$ spots detectable at $\operatorname{span}=10$, respectively, as seen in Supplementary Table S1). One may consider different thresholding or counting algorithms to of large median filter residuals (e.g., $12 / 16$ ) is sufficient evidence for classifying a concentration as positive. The trade-off of requiring a high proportion of residuals to be large is that the false positive rate is reduced but the false negative rate increases, and vice versa. determine whether a given proportion 
Table 1 also shows the results of the median filter as compared to two other methods for spot detection. We used the popular SAM method (11) and a Dunnett's $t$-test (12), both adjusted for multiple comparisons. For these methods, we compared each well's 16 observations versus the 576 observations in the buffer-only area, rows B-D. After subtracting background from foreground, we identified the wells where the mean intensities were significantly different from the control intensities. The sensitivities and specificities of each of these approaches appear in Table 1. The SAM method performed inefficiently compared to the median filter and the controlled Dunnett's $t$-test. The Dunnett's $t$-test and the median filter performed comparably, with the median filter making more false positive errors and the Dunnett's $t$-test making more false negative errors.

With the small number of spots that we considered for our four macroarray experiments, we were able to assess the spots individually. Visual inspection of each spot would be unfeasible when dealing with the 100,000 spots typically used in microarray experiments. An advantage of the median filter method is that the spots are quantified in terms of how unusual they are, compared to the immediate neighborhood around them. Large median filter residuals correspond with a large, unusual signal. We had 16 replicates per well, and the quantification could be refined to include a vote-counting scheme where a majority of spots at the same concentration would have to be above the detection threshold to be considered present.

The threshold at which an algorithm would classify the residuals as either present or absent is dependent on the experiment, the median filter smoothing span, and one's tolerance for misclassification. The detection threshold may be visualized by drawing horizontal lines across the needle plot shown in Figure 2. Lines that extend above a given horizontal line would correspond to detectable signals. The risk of setting the threshold too low is the inflation of the false positive rate. We chose our thresholds empirically by applying a partitioning model after visually classifying the wells as detectable or not detectable. When visual classification is not feasible, one may consider using a threshold based on the maximum of the residuals in control wells where no material was present. Alternatives to threshold detection may include smoothing the residuals with, for example, a running maximum and a large span $(13,14)$.

Despite the availability of many high-throughput solutions for protein arrays, there are still many applications in which more manual procedures are necessary. These procedures require tailored statistical and numerical analysis techniques for analysis. In our scenario, we applied a convenient and intuitive method for detecting present calls in images where the background noise was correlated with the foreground signal. The median filter approach performed as well or better than visual inspection and a controlled Dunnett's $t$-test, and it had the advantage of quantifying image data that could then be passed to more advanced statistical techniques.

\section{ACKNOWLEDGMENTS}

The authors would like to thank YateChing Yuan (City of Hope Informatics Core), Jim Bolen, and Lucy Brown (City of Hope Analytical Cytometry Core). The authors are also grateful for the valuable input and lively discussions with Dr. Robert Nathan.

\section{COMPETING INTERESTS STATEMENT}

The authors declare no competing interests.

\section{REFERENCES}

1. Grubb, R.L., V.S. Calvert, J.D. Wulkuhle, C.P. Paweletz, W.M. Lineham, J.L. Phillips, R. Chuaqui, A. Valasco, et al. 2003. Signal pathway profiling of prostate cancer using reverse phase protein arrays. Proteomics 3:2142-2146

2.Liotta, L.A., V. Espina, A.I. Mehta, V. Calvert, K. Rosenblatt, D. Geho, P.J. Munson, L. Young, et al. 2003. Protein microarrays: meeting analytical challenges for clinical applications. Cancer Cell 3:317-325.

3. Robinson, W.H., C. DiGennaro, W. Hueber, B.B. Haab, M. Kamachi, E.J. Dean, S. Fournel, D. Fong, et al. 2002. Autoantigen microarrays for multiplex characterization of autoantibody responses. Nat. Med. 8:295-301.

4. Yan, F., A. Sreekumar, B. Laxman, A.M. Chinnaiyan, D.M. Lubman, and T.J. Barder. 2003. Protein microarrays using liquid phase fractionation of cell lysates. Proteomics 3:1228-1235.
5. Madoz-Gurpide, J., H. Wang, D.E. Misek, F. Brichory, and S.M. Hanash. 2001. Protein based microarrays: a tool for probing the proteome of cancer cells and tissues. Proteomics 1:1279-1287.

6.Zhou, Y.-X., P. Kalocsai, J.-Y. Chen, and S. Shams. 2000. Information processing issues and solutions associated with aicroarray technology, p. 167-200. In M. Schena (Ed.), Microarray Biochip Technology. Eaton Publishing, Natick, MA.

7. Tukey, J.W. 1985. The Collected Works of John W. Tukey. D.R. Brillinger (Ed.). Wadsworth Advanced Books \& Software, Belmont, CA, p. 837-855.

8. Gonzales, R.C. and R.E. Woods. 2002. Digital Image Processing. Prentice-Hall, Englewood Cliffs, NJ.

9.Justusson, B.I. 1981. Two-Dimensional Digital Signal Processing II, Transforms and Median Filters. T.S. Huang (Ed.). SpringerVerlag, New York, p. 161-196.

10. Moore, A.W., Jr. and J.W. Jorgenson. 1993 Median filtering for removal of low-frequency background drift. Anal. Chem. 65:188-191.

11. Tusher, V., R. Tibshirani, and G. Chu. 2001 Significance analysis of microarrays applied to the ionizing radiation response. Proc. Natl. Acad. Sci. USA 98:5116-5121.

12. Dunnett, C.W. 1955. A multiple comparison procedure for comparing several treatments with a control. J. Am. Stat. Assoc. 50:1096-1121.

13. Thisted, R.A. 1988. Nearest-neighbor estimates, p. 342. In Elements of Statistical Computing, section 6.1.4. Chapman and Hall, New York.

14. Hastie, T.J. and R. Tibshirani. 1990 Smoothing in detail, p. 39-78. In Generalized Additive Models, ch. 3. Chapman and Hall, London.

Received 28 November 2005; accepted 21 April 2006.

Address correspondence to David D. Smith, Department of Biostatistics, City of Hope National Medical Center, 1500 E Duarte Rd, Duarte, CA 91010, USA. e-mail:dsmith02@coh.org.

To purchase reprints of this article, contact: Reprints@BioTechniques.com 